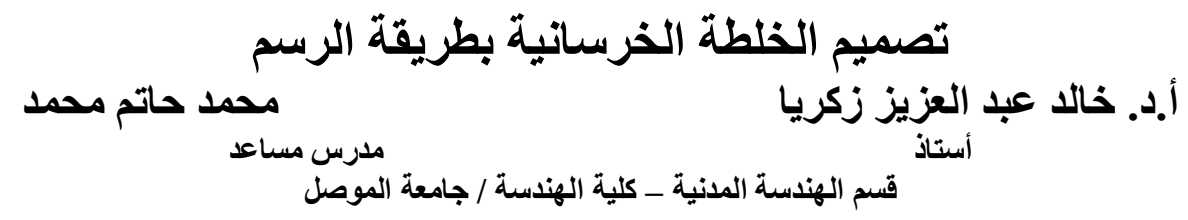

\title{
الخلاصة
}

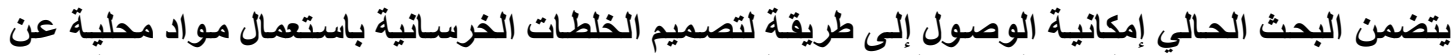

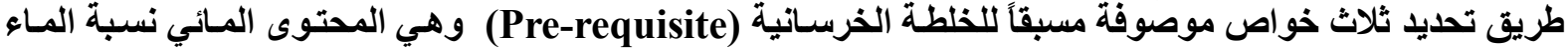

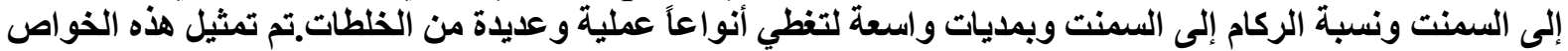

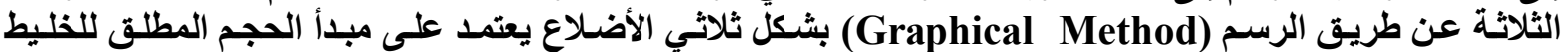

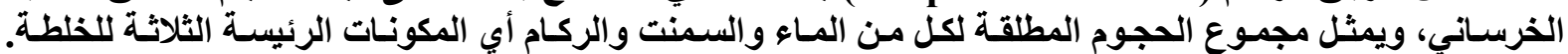

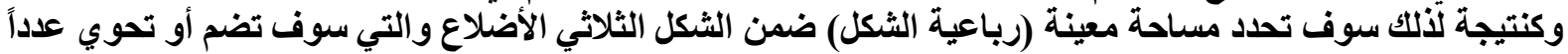

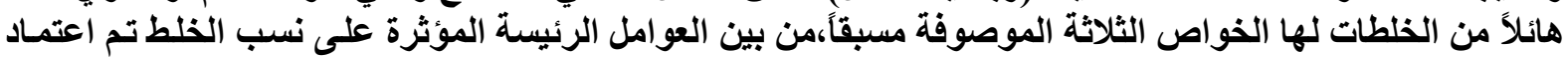
الآتي في البحث الحالي وكما يلي: الئي:

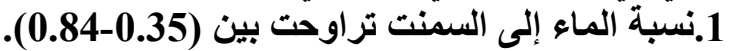

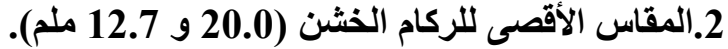

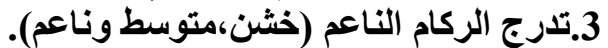

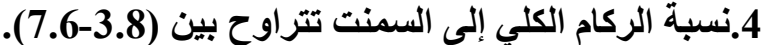

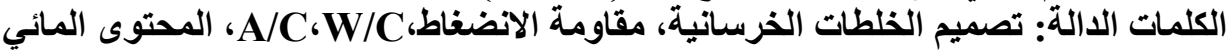

\section{Graphical Method of Concrete Mix Design}

\author{
Dr. Khalid A. Zakaria \\ Professor
}

\section{Mohammad Hatem Mohammad Assint lecturer}

Civil Eng. Dept. College of Engineering, University of mosul

\begin{abstract}
The current research work caters for the possibility of arriving at a method for designing concrete mixes (job mix) using locally available materials by means of three prescribed properties of the mix as design pre-requisites, that is water content, water : cement ratio and aggregate : cement ratio of wide ranges to cover a good variety of practical mixes. The above mentioned three prescribed properties can be represented in a graphical solution manner using a trilinear diagram depending on the fact that the absolute volume of the concrete mixture consisting of three materials, cement, water and aggregate is the sum of absolute volumes of the three materials. As a result and from this representation a quadrilateral area can be formed which should include a wide range of mixes having all the prescribed properties mentioned above. The current work will consider the following main influencing parameters on the job mix:
\end{abstract}

1. Water : cement ratios between $0.35-0.84$.

2. Maximum aggregate sizes of 20 and $12.7 \mathrm{~mm}$.

3. Fine aggregate ranges of fine, medium and coarse (B.S 882-1992 [11])

4. Aggregate : cement ratios between 3.8-7.6.

Key words: Concrete mix design, Compressive strength, W/C, A/C, Water demand

قبل: 2010 - 6

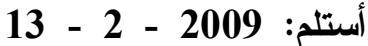




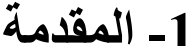

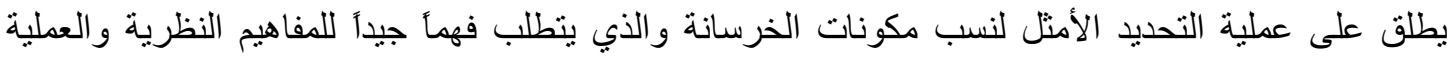
ودراسة خو اص المواد المحلية والتطبيقات المطلوبة منها ( بتصميم الخليط الخرساني) فالخواص المطلية الخية والمانية المحددة للخليط الخرساني تتضمن مايلي: خو اص الخرسانة الطرية. الخو اص الميكانيكية المطلوبة للخرسانةِ المئصَلَبِةِ منل متطلباتِ الديمومة و المقاو مة.

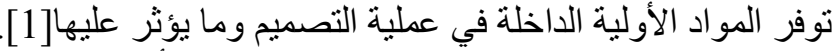

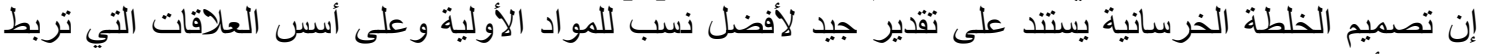

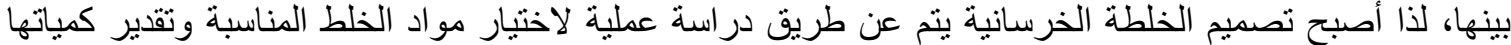

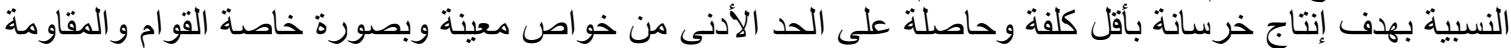

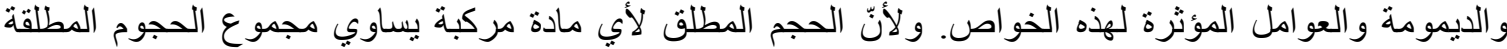

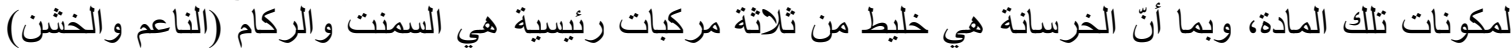

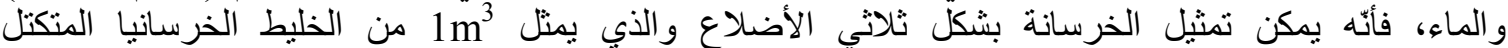

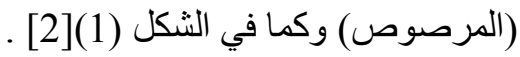

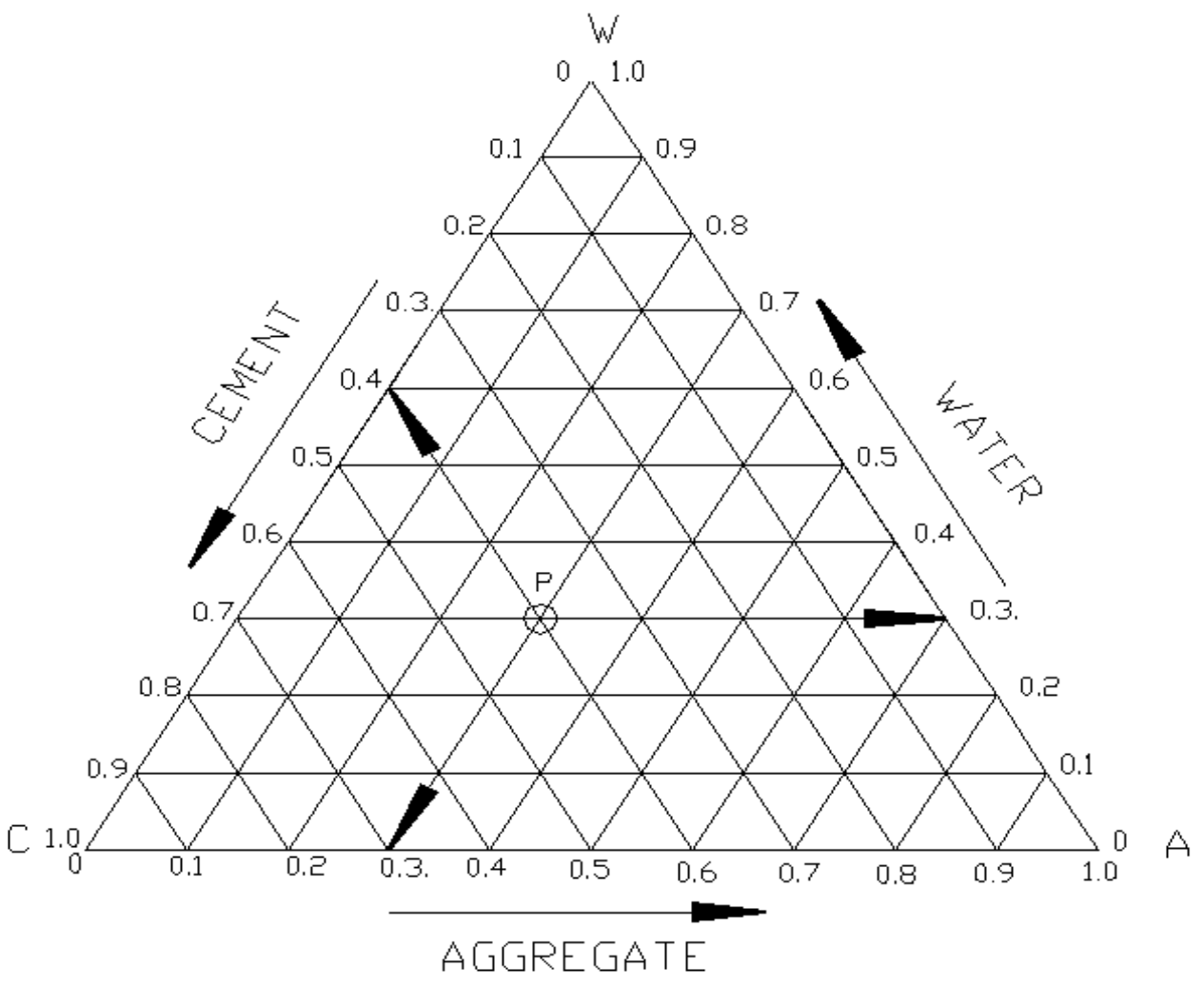

الشكل(1): حجم 1م ${ }^{3}$ من خليط يتكون من (السمنت ،الماء والركام)[2].

يمثل المتلث (WCA) منر مكعب و احد من خلبط متكتل من الماء و السمنت و الركام (ناعم+خشن)، فقي النقطة

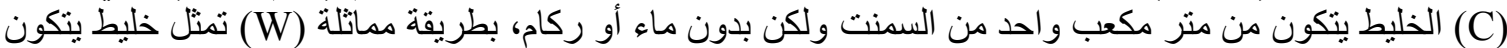

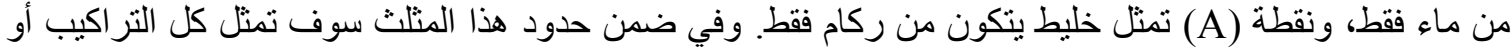

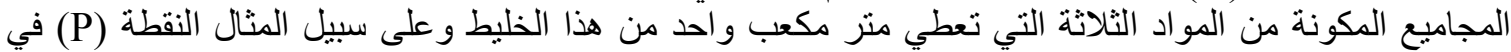

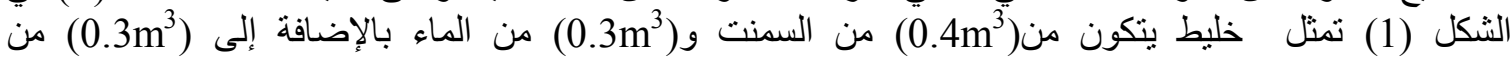

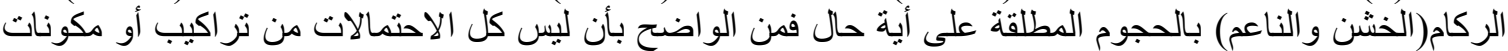

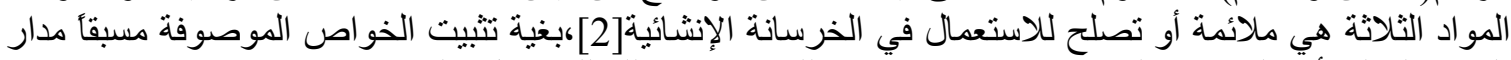

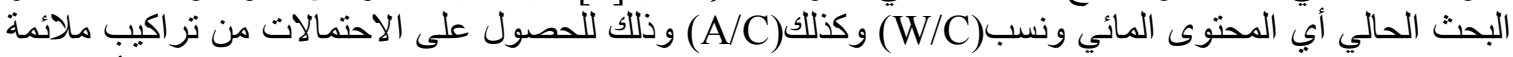

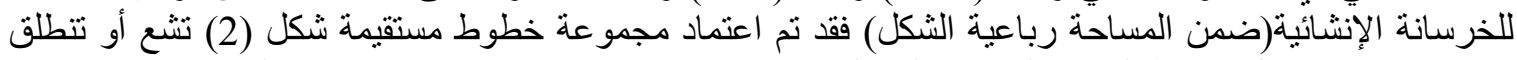

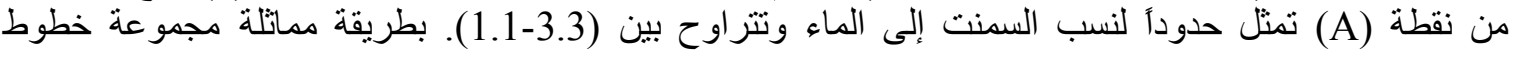




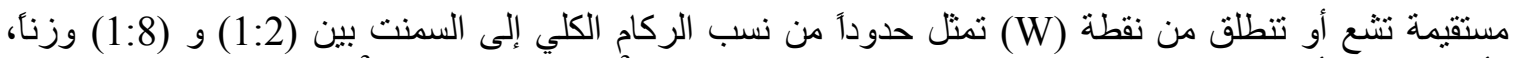

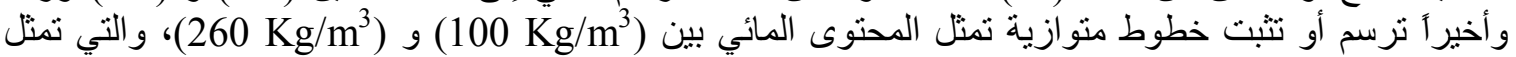

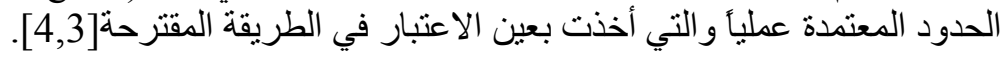

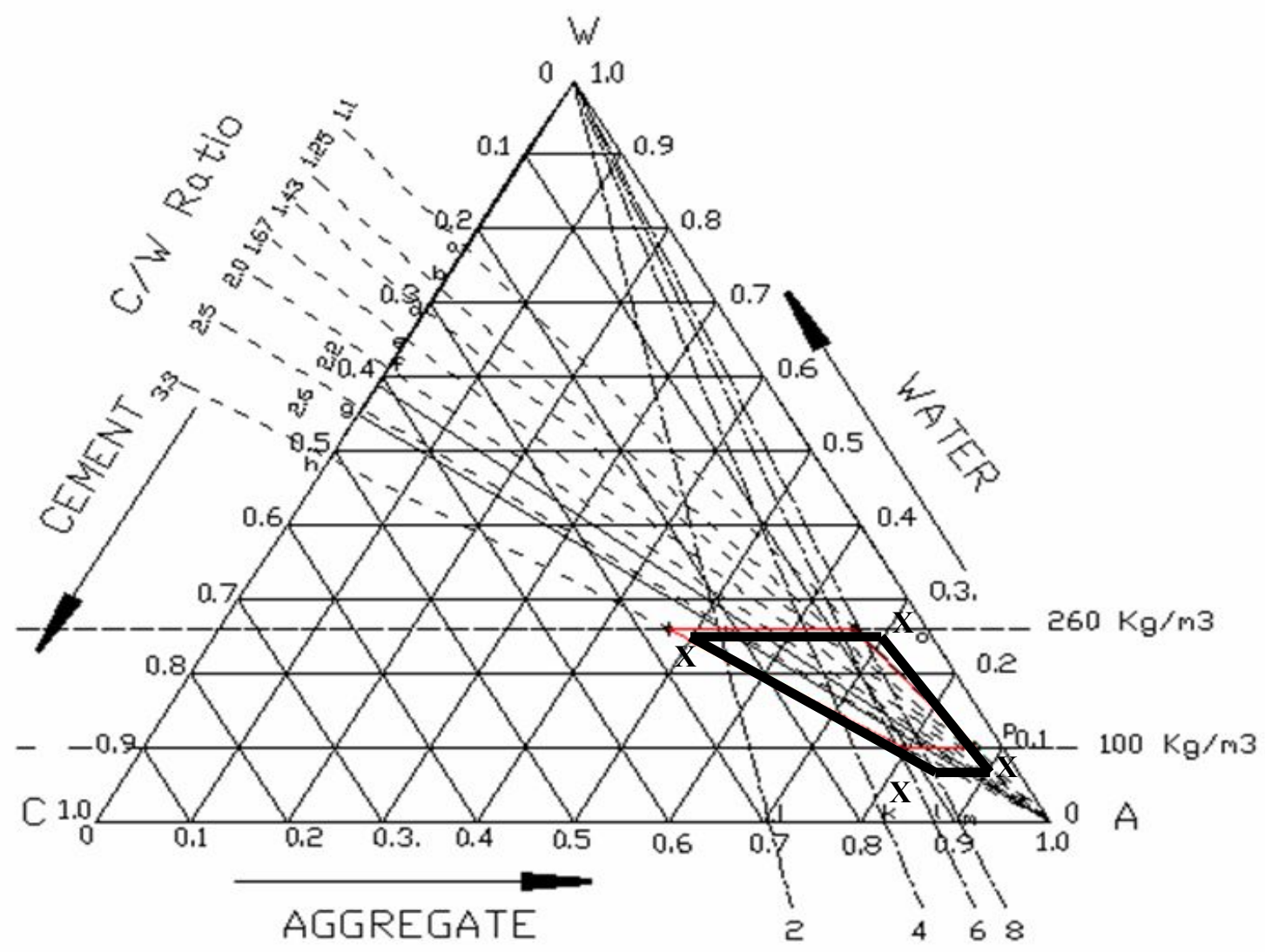

الشكل(2): مبادئ تصميم الخلطة الخرساتية[2].

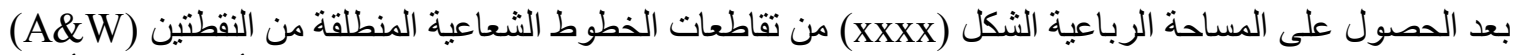

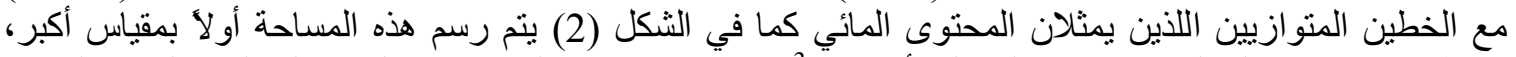

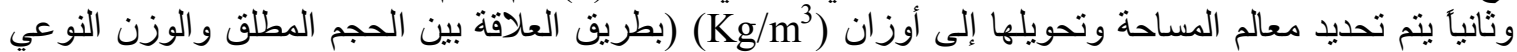

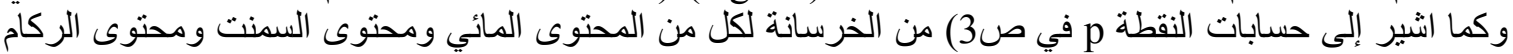

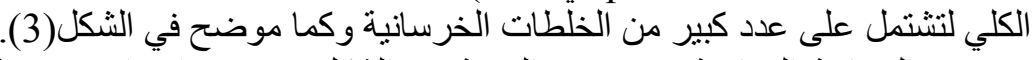

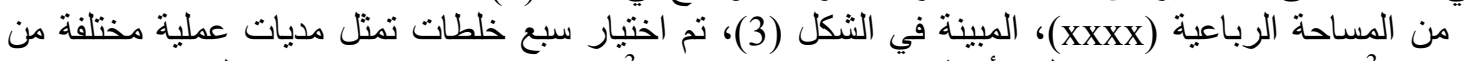
السمنت (250 Kg/m (1978-1882 Kg/m³)

الجدول(1): الخلطات الخرسانية المعتمدة في البحث الحالي .

\begin{tabular}{|c|c|c|c|c|c|}
\hline $\begin{array}{l}\text { A/C نسبة الوزنية } \\
\text { ن }\end{array}$ & $\begin{array}{l}\text { W/C نسبة الوزنية } \\
\text { نوزية }\end{array}$ & $\begin{array}{l}\text { الركام الكلي } \\
\left(\mathrm{Kg} / \mathrm{J}^{3}\right)\end{array}$ & $\begin{array}{l}\text { المحتوى المائي } \\
\left(\mathrm{Kg} / \mathrm{m}^{3}\right)\end{array}$ & $\begin{array}{l}\text { محتوى السمنت } \\
\left(\mathrm{Kg} / \mathrm{m}^{3}\right)\end{array}$ & الخرسسانية الخلطات \\
\hline 7.6 & 0.84 & 1900 & 210 & 250 & الخلطة 1 \\
\hline 7.2 & 0.70 & 1908 & 186 & 265 & الخلطة 2 \\
\hline 6.9 & 0.45 & 1978 & 128 & 285 & الخلطة 3 \\
\hline 6.5 & 0.54 & 1939 & 163 & 300 & الخلطة 4 \\
\hline 6.2 & 0.60 & 1957 & 189 & 315 & الخلطة 5 \\
\hline 4.9 & 0.51 & 1882 & 198 & 387 & الخلطة 6 \\
\hline 3.8 & 0.35 & 1885 & 173 & 496 & الخلطة 7 \\
\hline
\end{tabular}




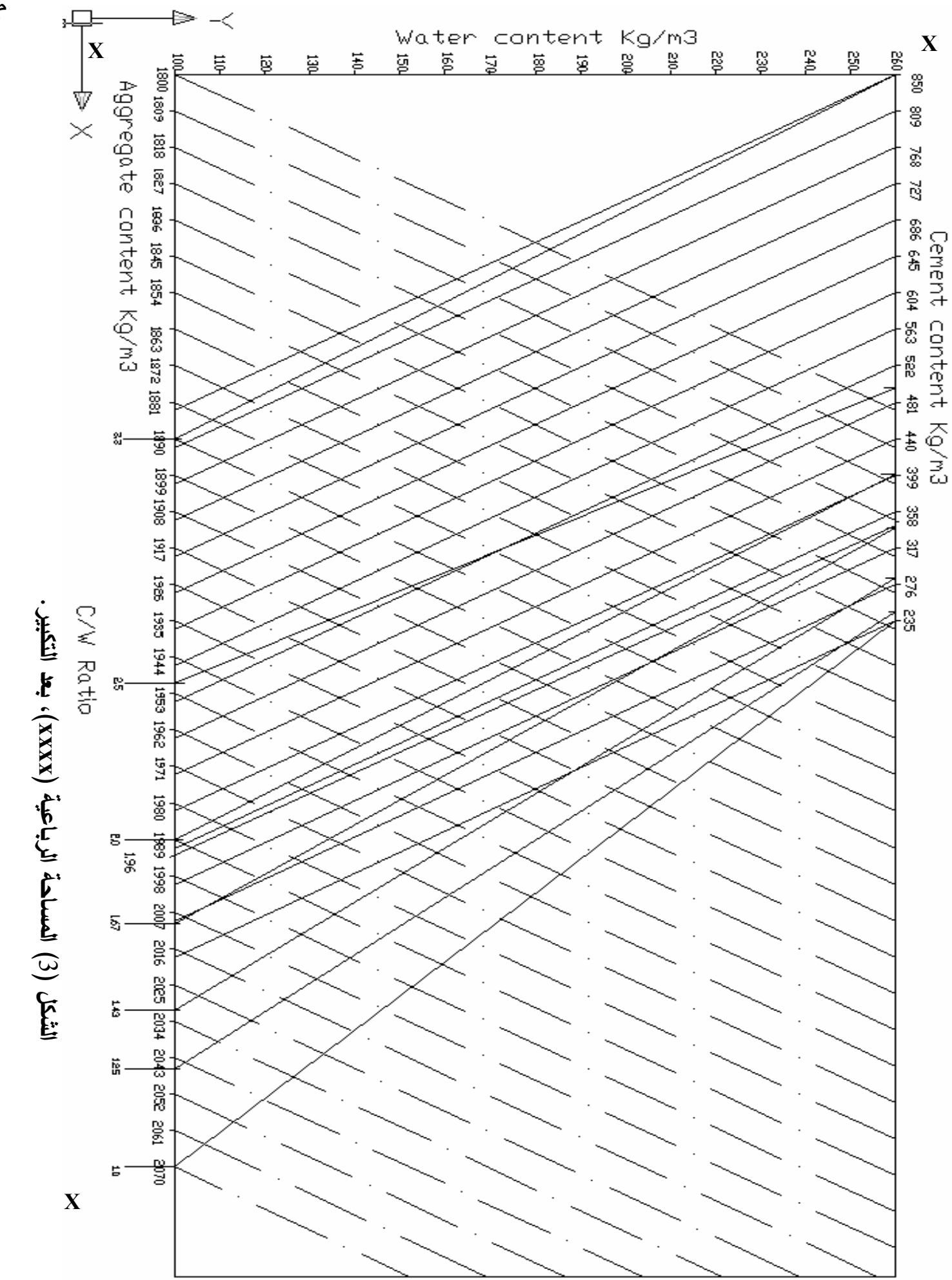

و يلاحظ من الجدول أعلاه بأن مديات المحتوى المائي (260) و (210-128 Kg/m) هي ضمن المحتوى المائي الموصوف

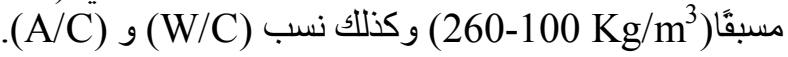




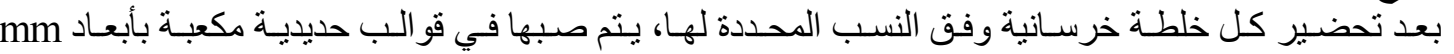

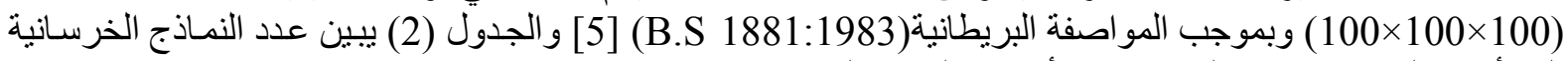
التي أعدت لغرض دراسة الخصائص الأساسية المشار إليها.

الجدول(2): عدد النماذج الخرسانية المطلوبة لكل خلطة .

\begin{tabular}{|c|c|c|c|c|}
\hline عدد النماذج & عمر الفحص) & الخقاس الأقصى للركام & نوع الرعام & الخلطة الخرسانية \\
\hline 3 & 28 & 20 & رمل خشن & \multirow[t]{6}{*}{ M1 } \\
\hline 3 & 28 & 20 & رمل متوسط & \\
\hline 3 & 28 & 20 & رمل ناعم & \\
\hline 3 & 28 & 12.7 & رمل خشن & \\
\hline 3 & 28 & 12.7 & رمل متوسط & \\
\hline 3 & 28 & 12.7 & رمل ناعم & \\
\hline
\end{tabular}

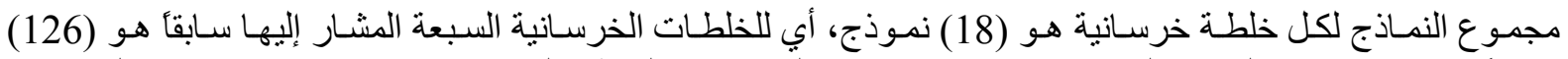

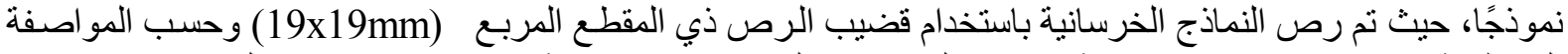

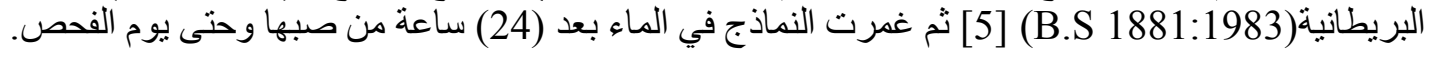

2-2-2 خواص المواد المستخدمة في الاراسة العملية:

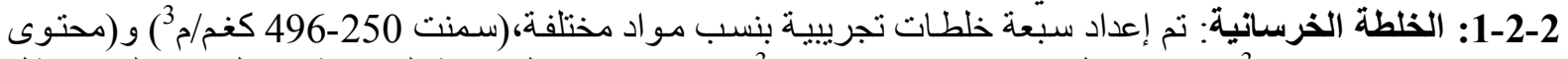

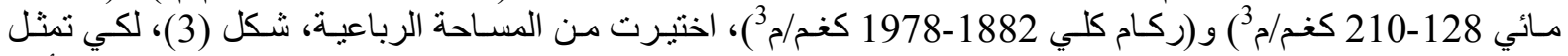

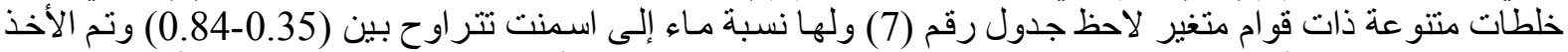

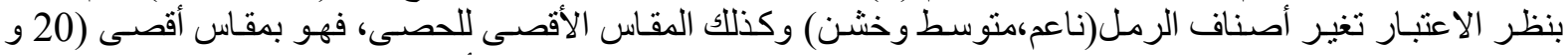

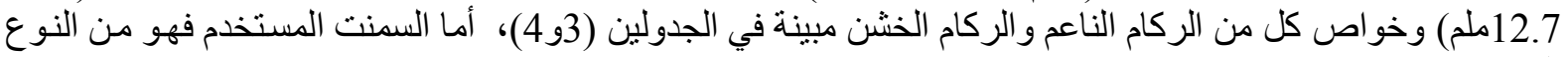

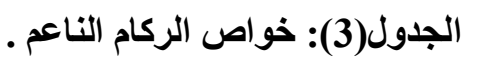

البورتلاندي الاعتيادي (O.P.C).

\begin{tabular}{|c|c|c|c|c|}
\hline \multirow{2}{*}{ 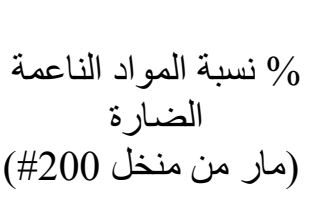 } & \multirow{2}{*}{ الامتصاص } & \multicolumn{2}{|c|}{ الوزن النوعي } & \multirow{2}{*}{ نوع الركام الناعم } \\
\hline & & Oven dry & S.S.D & \\
\hline 1.9 لا يزيد عن 5\% & 2.47 & 2.56 & 2.63 & الرمل الخشن \\
\hline 1.9 لا يزيد عن 5\% & 2.50 & 2.54 & 2.61 & الرمل المتوسط \\
\hline 1.9 لا يزيد عن 5\% & 2.54 & 2.52 & 2.6 & الرمل الناعم \\
\hline
\end{tabular}

الجدول(4): بعض خواص الركام الخشن ذات الصلة .

\begin{tabular}{|c|c|c|c|}
\hline \multirow[t]{2}{*}{ الامنصاص \% } & \multicolumn{2}{|c|}{ الوزن النوعي } & \multirow[t]{2}{*}{ نوع الركام الخشن } \\
\hline & Oven dry & S.S.D & \\
\hline 1.000 & 2.62 & 2.66 & حصى بمقاس أقصى (12.7 mm) \\
\hline 0.930 & 2.62 & 2.66 & حصى بمقاس أقصىى (14 mm) \\
\hline 0.807 & 2.62 & 2.66 & حصى بمقاس أقصىى (20 mm) \\
\hline 0.807 & 2.62 & 2.66 & حصى بمقاس أقصى \\
\hline
\end{tabular}




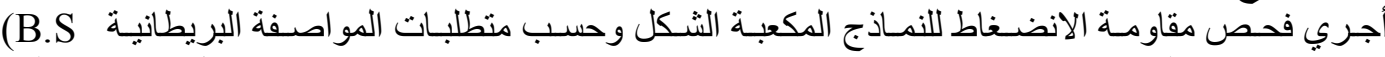

2-2-2

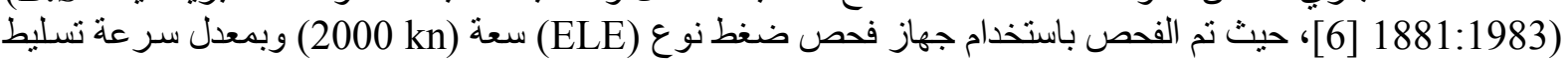

للحمل ( ) (3kn/s).

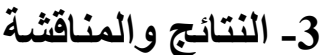

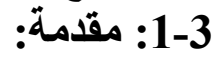

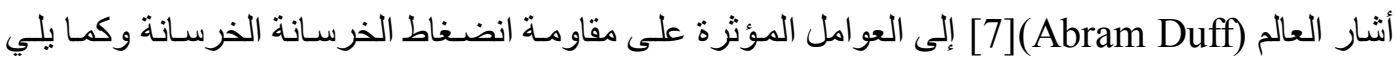

(لنفس المو اد وظروف الفحص فإن مقاومة انضـغاط الخرسـانة المتكتلة ولعمر معين تعتمد فقط على نسبة المـاء/السمنت

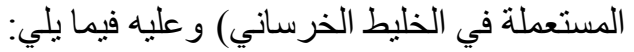

\section{2-3: تأثير نسبة الماء إلى السمنت (W/C ratio) على مقاومة الانضغاط:}

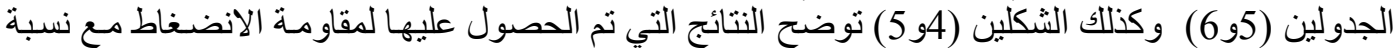

الماء إلى السمنت بعمر (28 يوم) لحصى بمقاس أقصى (5) (12.7و

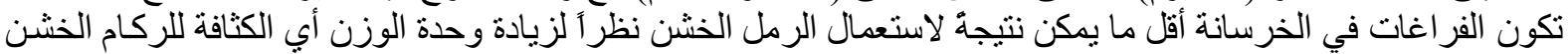

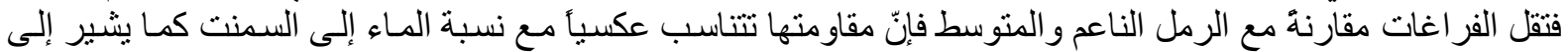

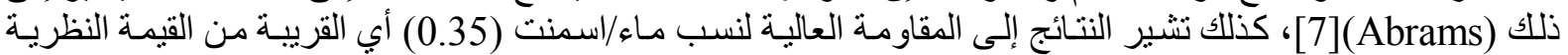

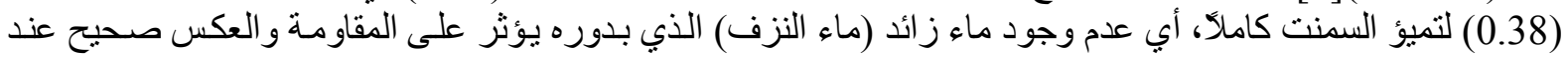

نسبة ماء/اسمنت (0.84) التمئ كالكان.

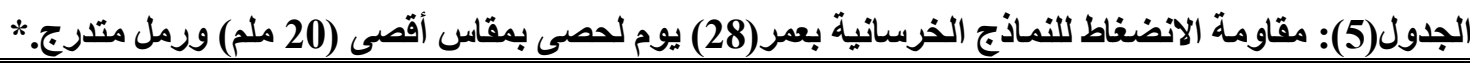

\begin{tabular}{|c|c|c|c|}
\hline \multicolumn{3}{|c|}{ مقاومة الانضغاط (نت/ملم2)- مقاس أقصى mm 20 للركام الخشن } & \multirow{2}{*}{$\begin{array}{l}\mathrm{W} / \mathrm{C} \\
\text { ratio }\end{array}$} \\
\hline رمل ناعم & رمل متوسط & رمل خشن & \\
\hline 54.2 & 57 & 60.0 & 0.35 \\
\hline 36.0 & 40.9 & 42.0 & 0.45 \\
\hline 32.5 & 32.8 & 35.0 & 0.51 \\
\hline 31.0 & 31.9 & 33.8 & 0.54 \\
\hline 23.4 & 24.8 & 26.3 & 0.60 \\
\hline 15.5 & 16.2 & 17.8 & 0.70 \\
\hline 10.5 & 10 & 11.0 & 0.84 \\
\hline
\end{tabular}

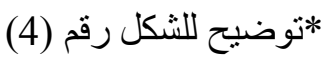

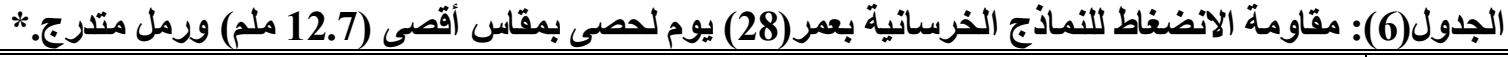

\begin{tabular}{|c|c|c|c|}
\hline \multicolumn{3}{|c|}{ مقاومة الانضغاط (نت/ملم2)- مقاس أقصى mm 12.7 للركام الخشن } & \multirow{2}{*}{$\begin{array}{l}\mathrm{W} / \mathrm{C} \\
\text { ratio }\end{array}$} \\
\hline رمل ناعم & رمل متوسط & رمل خشن & \\
\hline 58.0 & 59.3 & 63.0 & 0.35 \\
\hline 37.6 & 40.0 & 40.6 & 0.45 \\
\hline 35.8 & 36.3 & 37.3 & 0.51 \\
\hline 33.9 & 34.6 & 35.6 & 0.54 \\
\hline 25.8 & 26.6 & 28.0 & 0.60 \\
\hline 18.3 & 19.2 & 20.1 & 0.70 \\
\hline 12.1 & 12.7 & 14 & 0.84 \\
\hline
\end{tabular}

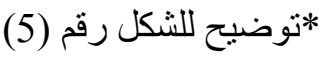




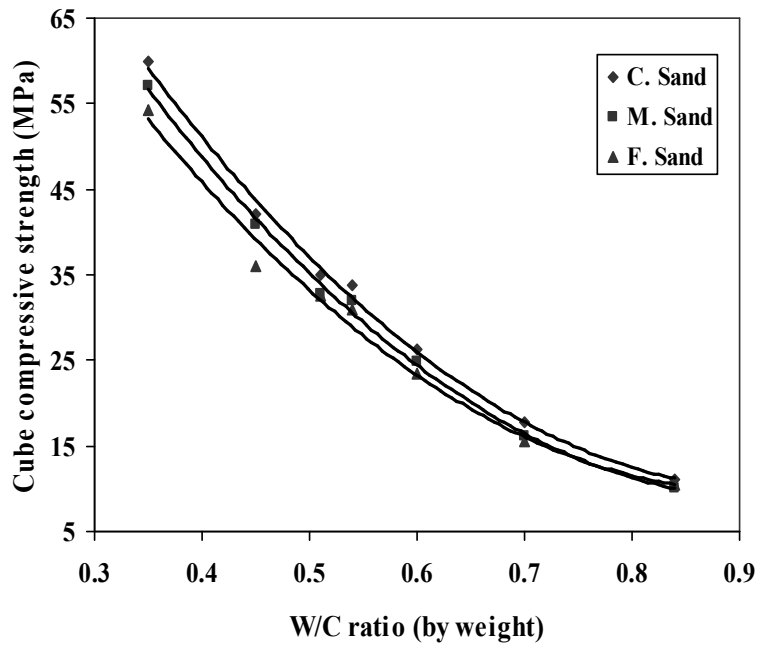

الثكل(4): نسبة الماء إلى السمنت مع مقاومة

الانضغاط لمكبات بأبعاد ( 100 mm ) وبعمر (28) يوم لحصى بمقاس أقصى(20 mm) ورمل متدرج.

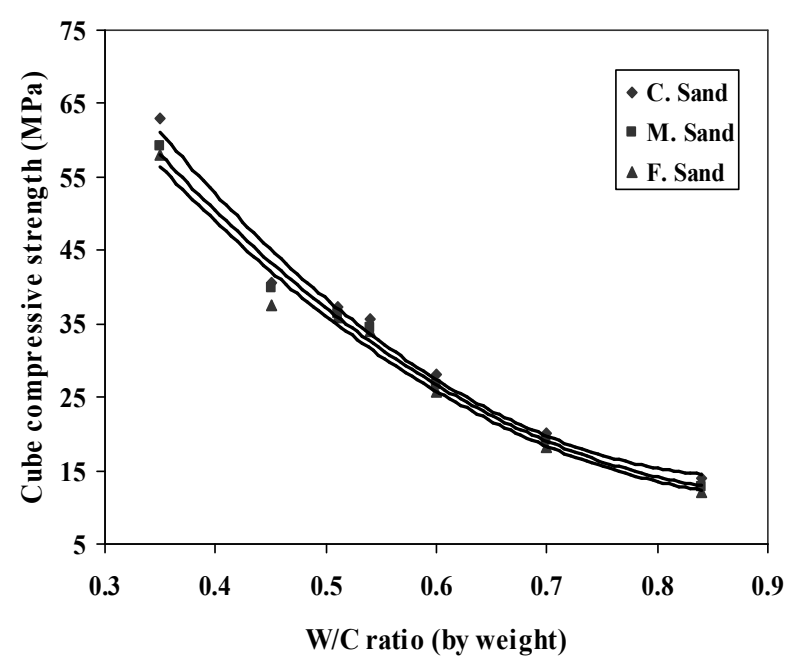

الثكل(5): نسبة الماء إلى السمنت مع مقاومة

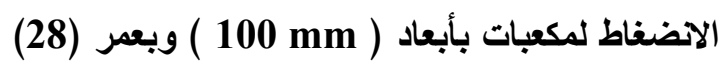
يوم لحصى بمقاس أقصى(12.7 mm) ورمل متدرج.

الجدول(7): تغاير الهطول مع نسب الماء إلى الاسمنت لحصى بمقاس أقصى (20 mm).

\begin{tabular}{|c|c|c|c|}
\hline \multicolumn{3}{|c|}{ الهطول (mm)- المقاس الأقصى 20 mm للركام الخشن } & \multirow{2}{*}{$\begin{array}{l}\text { W/C } \\
\text { ratio }\end{array}$} \\
\hline الرمل الناعم & الرمل المتوسط & الرمل الخشن & \\
\hline 9 & 10 & 19 & 0.35 \\
\hline 20 & 25 & 40 & 0.45 \\
\hline 32 & 37 & 52 & 0.51 \\
\hline 35 & 46 & 63 & 0.54 \\
\hline 44 & 53 & 72 & 0.60 \\
\hline 58 & 69 & 93 & 0.70 \\
\hline 80 & 100 & 130 & 0.84 \\
\hline
\end{tabular}

3-3-3: تأثير المقاس الأقصى للركام على مقاومة الاتضغاط:

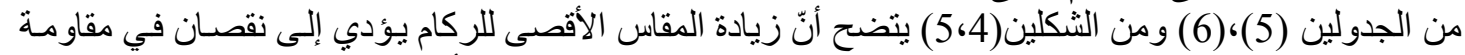

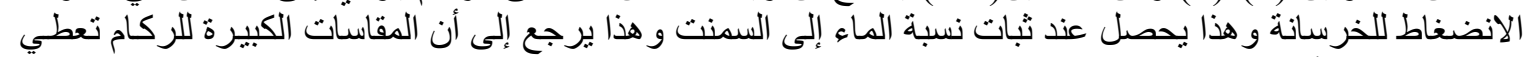

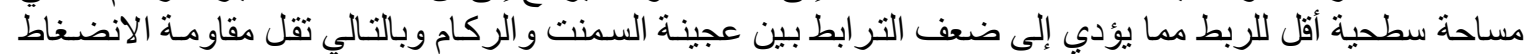
للخرسانة [10,9,8].

4-3: تأثير تدرج الركام الناعم على مقاومة الانضغاط:

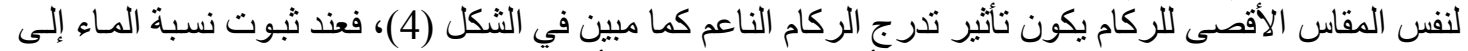

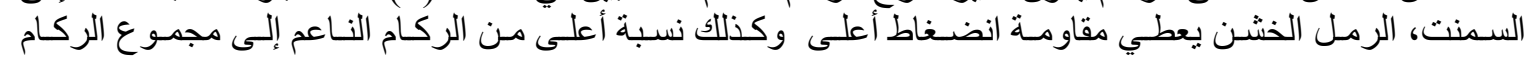

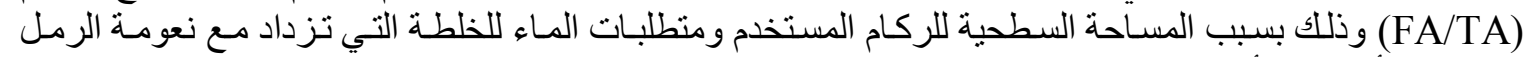
المستخدم (أي تكون أقل ما يمكن للرمل الخشن) وذللك لنفس مقاومة الانضغاط [10].

5-3: علاقة محتوى السمنت مع كل من نسبة الماء إلى السمنت (W/C ratio) ونسبة الركام الكلي إلى السمنت (A/C): 


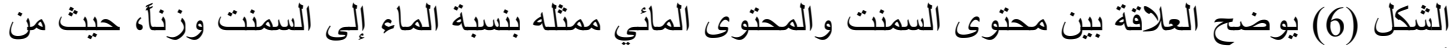

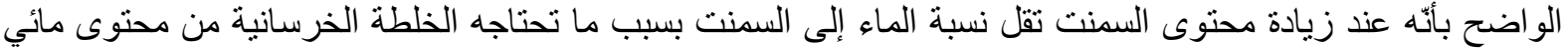

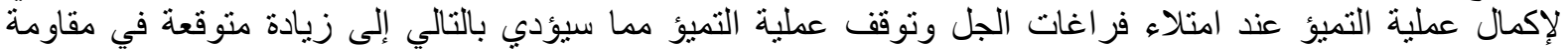

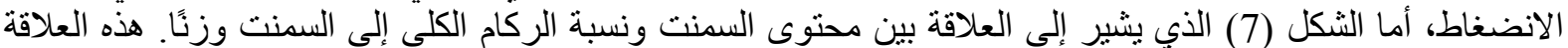

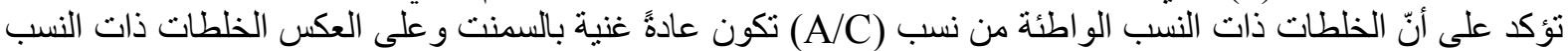

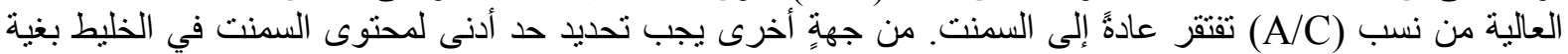

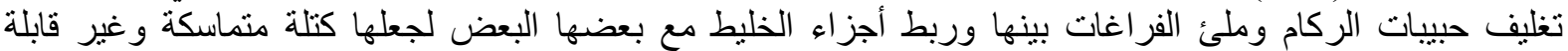

للانفصال وكتيمة بدرجة مقبولة [9,8].

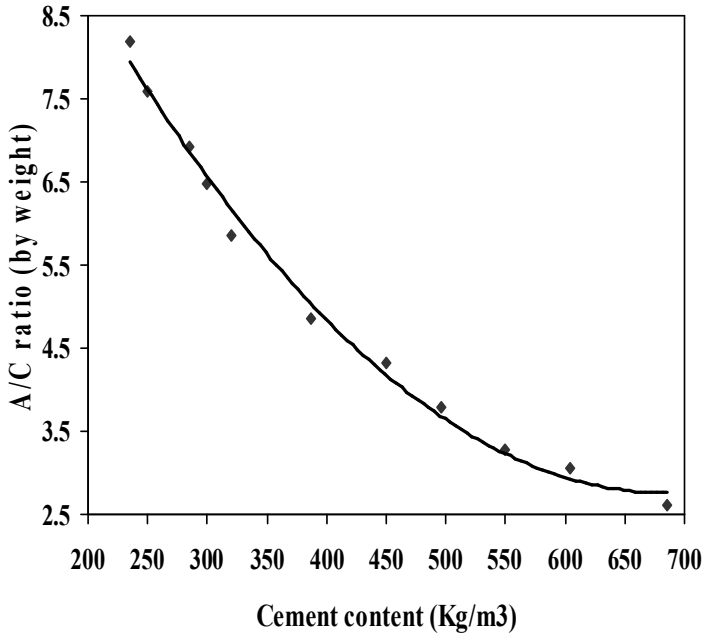

الشكل(6): العلاقة بين محتوى السمنت مع نسبة

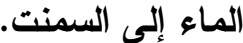

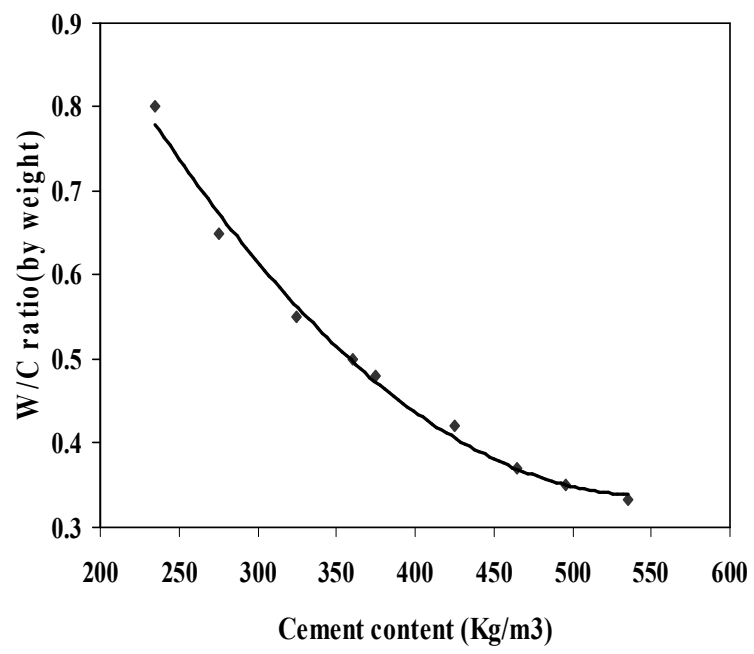

الثكل(7): العلاقة بين محتوى السمنت مع نسبة

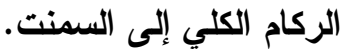

\section{6-3}

1-6-3 مثال تصميمي باستخدام العلاقات الناتجة عن المباتبة المساحة الرباعية:

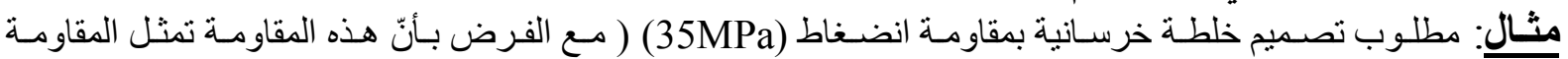

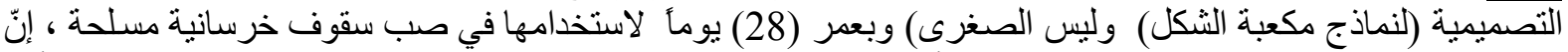

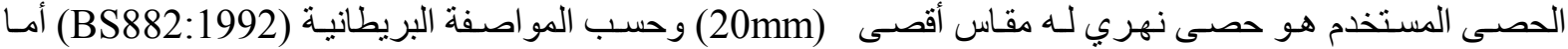

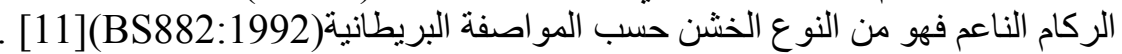

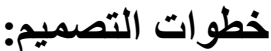
1- من الثكل (4) تحسب نسبة الماء إلى السمنت عند مقاومة الانضغاط التصميمية (35 MPa) باستخدام العلاقة

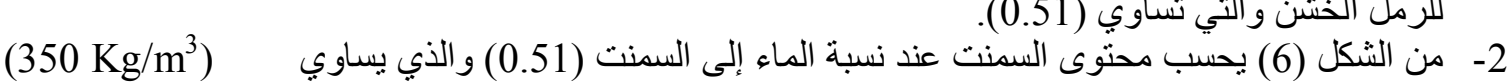

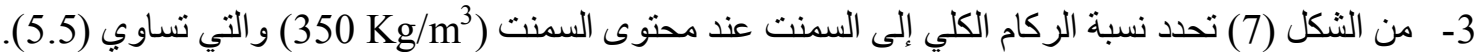

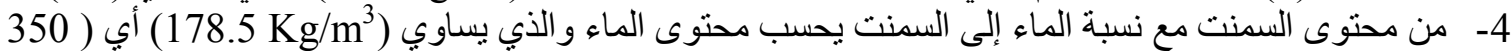
. 0.51

5- من محتوى السمنت مع نسبة الركام الكلي إلى السمنت يحسب محتوى الركام الكلي والذي يساوي ( 1925

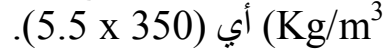

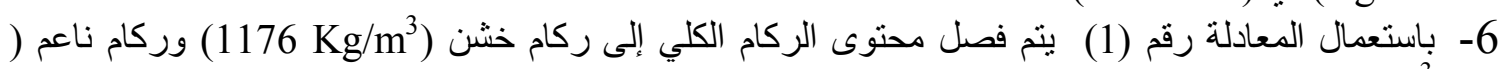
.[2] $\left(749 \mathrm{Kg} / \mathrm{m}^{3}\right.$ 


\section{$\mathrm{Z}-\mathrm{Y}$ \\ $A=\frac{Z-----1}{X-Z}$ [2] [13]}

: : نسبة الركام الناعم إلى الركام الخشن : A

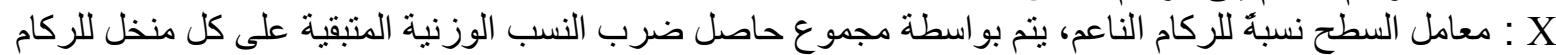

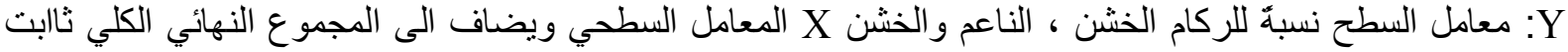

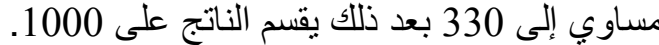

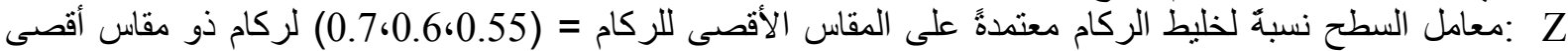
(10،20،40) ملم على التو الي.

Cement $=350 \mathrm{Kg} / \mathrm{m}^{3}$ أي إنّ الخلطة الخرسانية ستكون كالآتي

Water $=178.5 \mathrm{Kg} / \mathrm{m}^{3}$

Sand $=749 \mathrm{Kg} / \mathrm{m}^{3}$ G/S $=1176 / 749=1.57$

Gravel $=1176 \mathrm{Kg} / \mathrm{m}^{3}$

$$
1: 2.14: 3.36 / 0.51 \quad 3.36 / 2.14=1.57
$$

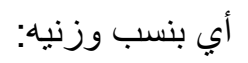

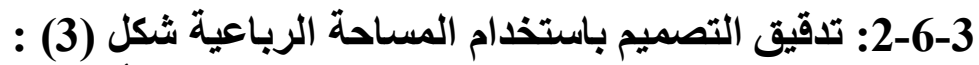

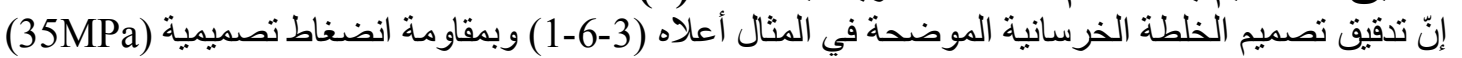
بعمر (28) يومًا كالآتي.

\section{خطوات تدقيق التصميم:}

1. من الثكل (4) تحسب نسبة الماء إلى السمنت عند مقاومة الانضغاط التصميمية (35 MPa) باستخدام العلاقة

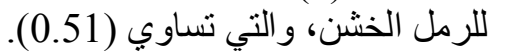

2. من الثكل (6) يحسب محتوى السمنت عند نسبة الماء إلى السمنت كما في الخطوة السابقة والذي يساوي ( 350 . $\left(\mathrm{Kg} / \mathrm{m}^{3}\right.$

3. من المساحة الرباعية ومن تقاطع نسبة السمنت إلى الماء (C/W=1.96) معكوس (0.51) ومحتوى السمنت

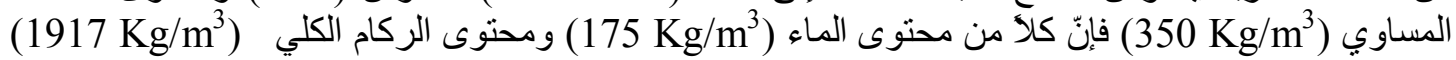
وكما في الثكل (8). (8).

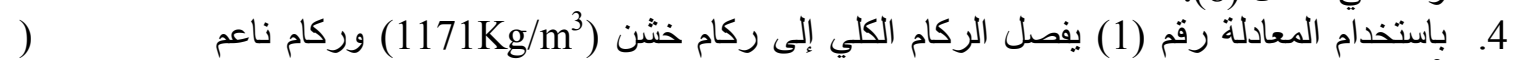

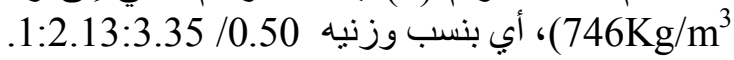

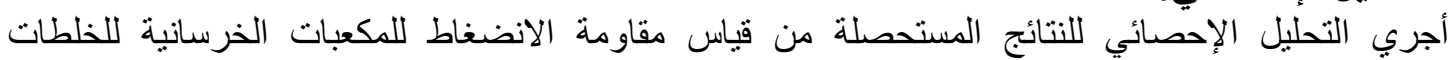
3-6-3

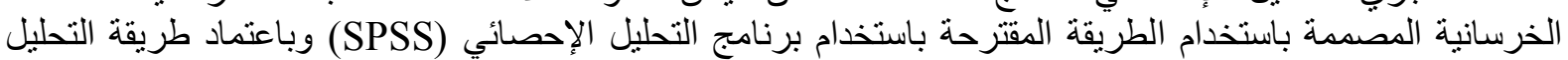

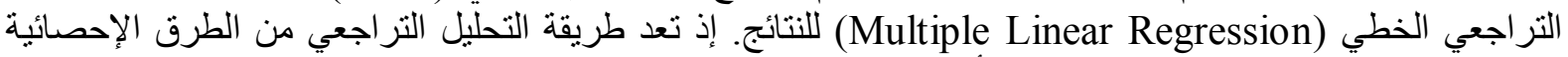

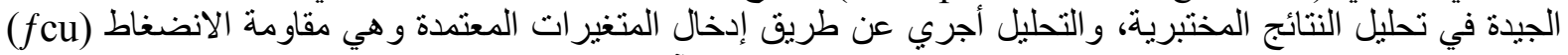

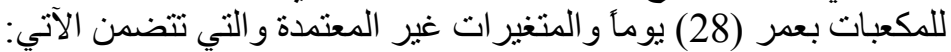
1. 2. ن نسبة الماء إلى السمنت (W/C ratio). 3. نسبة الركام الكلي إلى السمنت (A/C ratio).

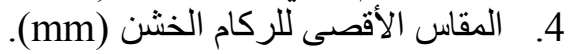

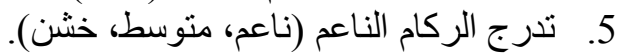

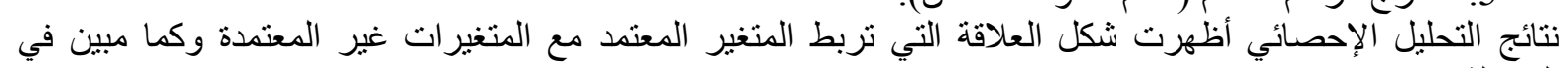
المعادلة رقم (2). (2) - (2)

$f c u=-58.208-70.968 \times W / C+0.207 \times C . C+10.258 \times A / C$ 


$$
\begin{aligned}
& \text { (fcu) : مقاومة الانضغاط بعمر (28) بومأ (MPa). } \\
& \text { (W/C) } \\
& \text { (A/C) (لسبة الركام الكلي إلى السمنت وزئًا. } \\
& \text { (C.C) }
\end{aligned}
$$

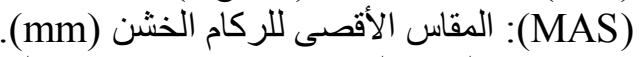

(F): تدرج الركام الناعم ويعوض بلركم الأمق 1 عن الرمل الناعم، رقم 2 عن الرمل المتوسط ورقم 3 عن الرمل الخشن

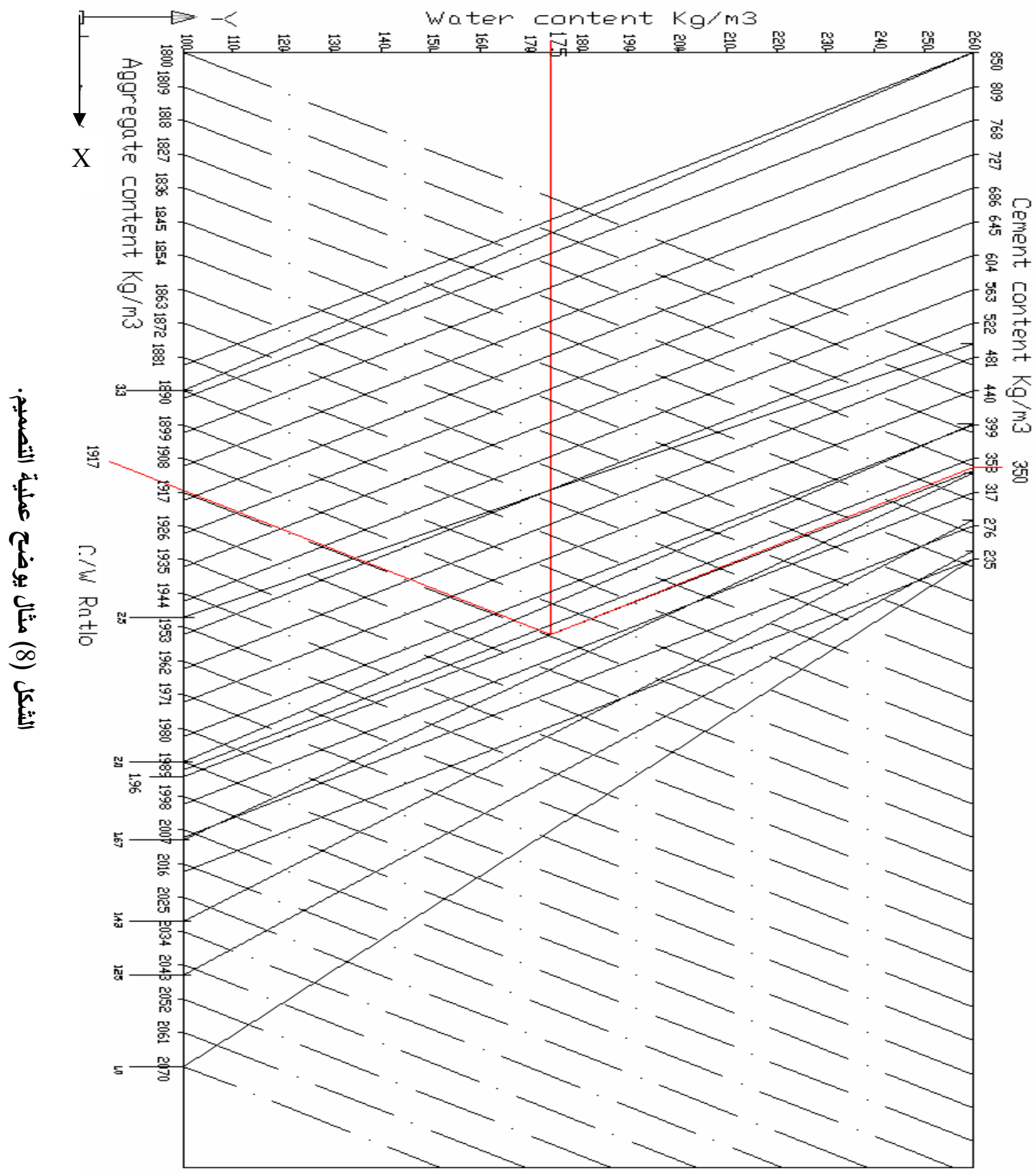




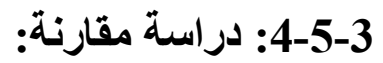

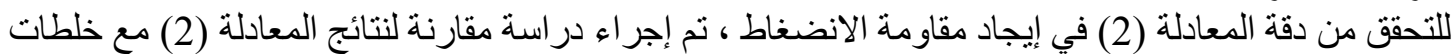

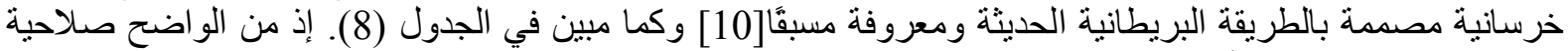

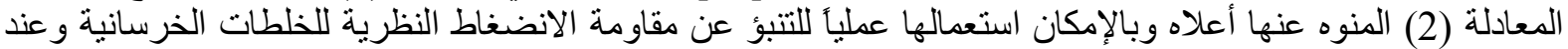

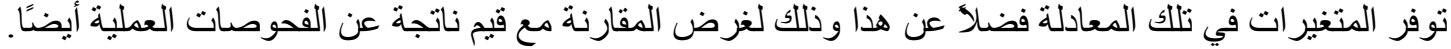

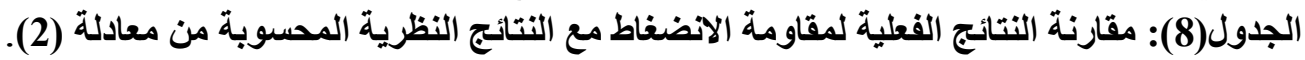

\begin{tabular}{|c|c|c|c|c|c|c|}
\hline $\begin{array}{c}\text { الانضاط } \\
\text { الفعلية } \\
\text { (MPa) } \\
\end{array}$ & 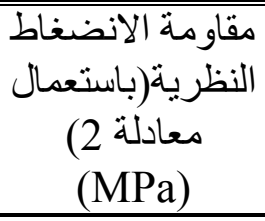 & ال الناعم & للركام الخثن (mm الأقصى & $\begin{array}{c}\text { محتوى السمنت } \\
(\mathrm{Kg} / \mathrm{m} 3)\end{array}$ & $\begin{array}{l}\mathrm{A} / \mathrm{C} \\
\text { ratio }\end{array}$ & $\begin{array}{l}\mathrm{W} / \mathrm{C} \\
\text { ratio }\end{array}$ \\
\hline $36.50[10]$ & 35.70 & خشن & 20 & 375 & 4.80 & 0.460 \\
\hline $46.10[10]$ & 44.50 & ناعم & 20 & 425 & 4.15 & 0.390 \\
\hline
\end{tabular}

\section{4- 4 الاستنتاجات}

1. إن طريقة تصميم الخلطات الخرسانية بطريقة الرسم كما يقترح البحث الحالي يجب أنسات أن تحدد بمجال ثلاثة

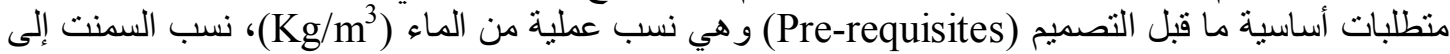

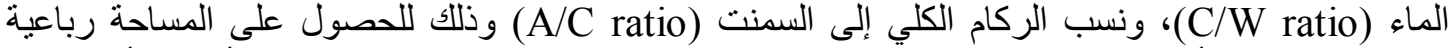
الثكل والتي تضم أو تحوي عدداً هائلاً من الخلطات الخرسانية ومن المفترض لهذه الخلطات أن تفي أو تتطابق

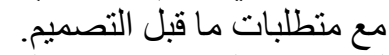

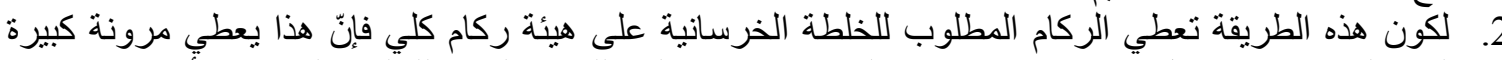

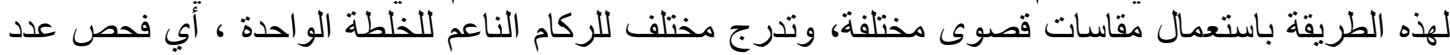

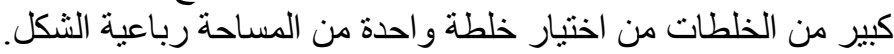

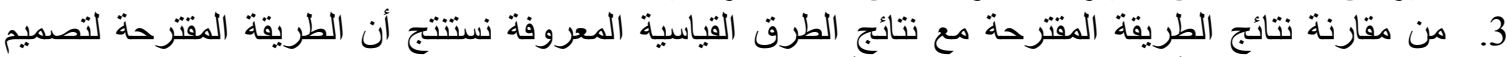

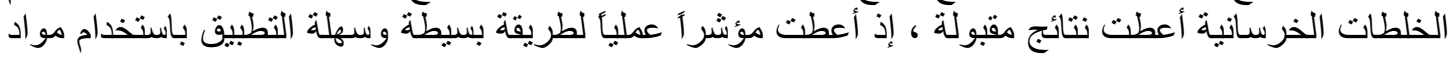

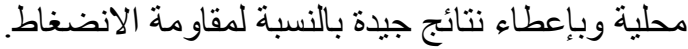

\section{5- التوصيات}

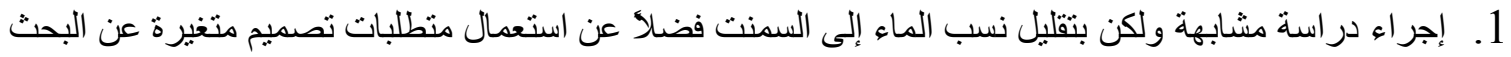
الحالي تحديدأ نسبة الركام الكلي إلى السمنت وكذللك استعمال النسب القليلة من السمنت إلى المـاء(w/c ratio) بغية إنتاج خرسانة عالية المقاومة المبة. 2.

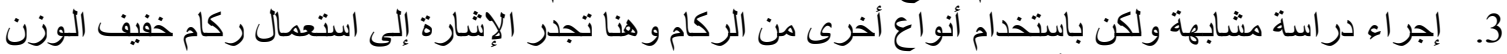

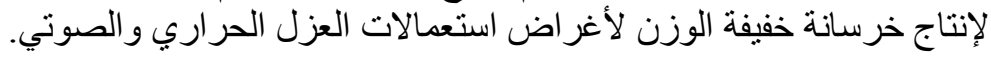

[1] Steven, H. Kosmatka, Beatrix, Kerkhoof, and William, C. Panarese, "Design and Control of Concrete Mixtures", Fourteenth Edition, Portland Cement Association, 2003,pp.149-177.

[2] Zakaria, Khalid A., and Waleed, A. Thanoun, "The Simplified Theory of Mix Design”, 5th Sci. Conf./SRC-Iraq, Baghdad, Vol.4, Part 1, 7-11 October, 1989.

[3] M. Simon, K. Snyder, and G. Frohusdorff, "Advances in Concrete Mixture Optimization", National Institute of Standards and Technology, London, September, 1999, pp. 21-32.

[4] Marcia, J. Simon, Eric, S. Lagergren, and Kenneth, A. Snyder, "Concrete Mixture Optimization Using Statistical Mixture Design Methods", 
International Symposium on High Performance Concrete, New Orleans, Louisiana, October, 1997, pp. 20-22.

[5] BS 1881: Part108: 1983 "Method For Making Test Cubes From Fresh Concrete", British Standard Institution, 1983.

[6] BS 1881: Part116: 1983 "Method For Determining of Compressive Strength of Concrete Cubes", British Standard Institution, 1983.

[7] Duff, M. Abrams, "Design of Concrete Mixtures", Structural Materials Research Laboratory, Bulletin, No. 1, May, 1919, pp. 1-20.

الخلف، مؤيد نوري و يوسف، هناء عبد، "تكنلوجيا الخرسانة"، وزارة التعليم العالي و البحث العلمي، الجامعة التكنولوجية، 1984

[9] Neville, A. M., "Properties of Concrete", 4th Edition, British Library, England, 2000.

العلو، غانم حسين قوجة، "الحد الأمثل للمحتويات الناعمة في الخرسانة وتأثيره على المقاومة و الكلفة"،

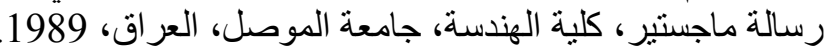

[11] BS 882-1992 "Aggregates From Natural Source For Concrete”, British Standard Institution, 1992.

الجادر ، محمد حاتم محمد، "تصميم الخلطة الخرسانبة بطريقة الرسم"، رسالة ماجستير، كلية الهندسة،

[13] Raju, N. Krishna, "Design of Concrete Mixes" College Booke store, 1701, Nai sarak , Delhi, India 1974.

تم اجراء البحث في كلية ألهندة = جامعة ألموصل 